\title{
Ultrafast multi-electron dynamics studied with THz-field streaking
}

\author{
$\underline{\text { M. Krikunova }}{ }^{1,2}$, E. Klimešová ${ }^{1}$, O. Kulyk ${ }^{1}$, T. Oelze ${ }^{2}$, B. Schütte ${ }^{3}$, T. Gebert ${ }^{4}$, \\ J. Andreasson ${ }^{1,5}$ \\ ${ }^{1}$ ELI Beamlines, Dolní Břežany by Prague, Czech Republic, maria.krikunova@eli-beams.eu \\ ${ }^{2}$ Technische Universität Berlin, Berlin, Germany \\ ${ }^{3}$ Max-Born Institute, Berlin, Germany, \\ ${ }^{4}$ Max Planck Institute for the Structure and Dynamics of Matter, Hamburg, Germany, \\ ${ }^{5}$ Chalmers University of Technology, Göteborg, Schweden
}

Temendous developments of novel ultrafast light sources based on High Harmonic Generation (HHG) or Free-Electron-Lasers (FELs) in the last decade have opened up new experimental regimes in the vacuum-ultraviolet (VUV), extreme-ultraviolet (XUV) down to X-ray spectral regions. Especially, the unique combination of coherent intense radiation with short pulse duration holds very promising perspectives for time-resolved molecular movies by obtaining information on molecular structure and function on atomic length scale with time resolution down to the attosecond domain. An understanding of the way how an intense X-ray light interacts with matter is of fundamental importance for studies of molecular structure and dynamics. Therefore this research topic is actively pursued by theoretical and experimental work within the photon science community.

The main objective of the Extreme Light Infrastructure - ELI-Beamlines project - in Dolní Břežany near Prague, Czech Republic is to establish an international user facility for fundamental and applied research with advanced laser sources. The research group for applications in molecular, biomedical, and material sciences works on the development of experimental capabilities using secondary sources that are driven by the unique ELI-Beamlines lasers. A central activity is the development of the multi-purpose user end-station (MAC) for experiments in atomic, molecular, and optical sciences and coherent diffractive imaging. The MAC user end-station is equipped with electron/ion time-of-flight spectrometers, Velocity Map Imaging detector, a detector for coherent diffractive imaging and state-of-the-art sample delivery systems to enable advanced photon science experiments on low density targets (atoms, molecules, clusters, nanometer size single intact free of substrate organic and inorganic particles and crystals, vacuum compatible tens of nanometers to a few micrometers thick liquid sheets formed with gas and by colliding jets technique, cylindrical GDVN jets and aerosols) in a broad range of the electromagnetic spectrum from $\mathrm{THz}$ to (soft) X-rays.

The THz-field driven streaking is a novel experimental technique to study the electronic response of many-electron systems to irradiation with intense ultra-short (soft) X-rays pulses [1]. The effect of lightfield streaking can be understood as an additional momentum acquired by the free electrons in the pres- ence of a dressing electric field [2]. By changing the time-delay between the ionizing XUV pulse with respect to the streaking field and measuring the electron kinetic energy spectra, a streaking spectrogram is obtained. In the streaking experiment half of the oscillation period of the streaking field has to be shorter than the temporal width of the electron distribution [2]. Therefore, to resolve the electron dynamics that is initiated by the femtosecond (soft) X-ray pulse, a THz-field is used [1].

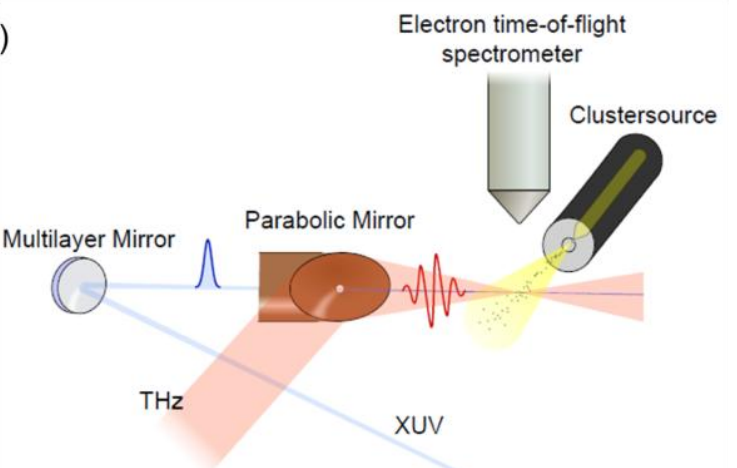

(b)

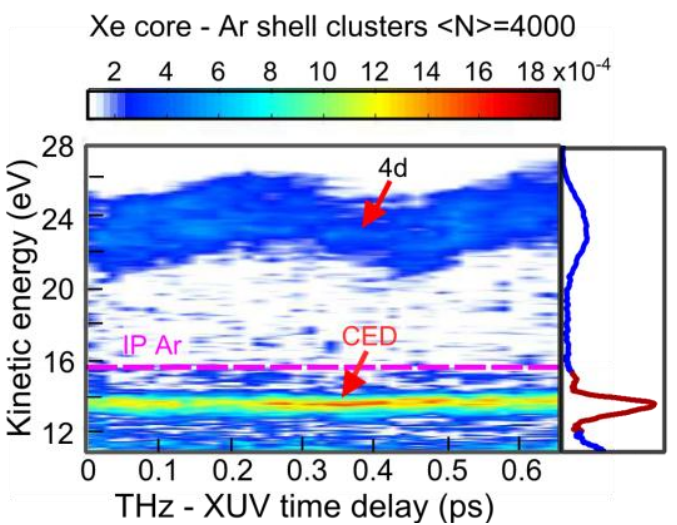

Fig. 1. (a) Principle of the THz-field driven streak-camera measurement. $\mathrm{THz}$ and XUV/(soft) X-ray pulses are collinearly overlapped and focused in the interaction region on atomic, molecular or nano-particle target. A series of photoelectron spectra, a so called streaking spectrogram, is measured with an electron-spectrometer as a function of relative time-delay between $\mathrm{THz}$ and XUV pulses. (b) $\mathrm{THz}-$ streaking spectrogram recorded in $\mathrm{Xe}$-Ar clusters at FreeElectron-Laser (FEL) in Hamburg, FLASH. An observation of an un-modulated peak (marked as CED) clearly discloses an electron emission delayed by at least several picoseconds after ionization of Xe-Ar clusters with intense FEL pulses. Data are reproduced from [3]. 
Figure 1 (a) shows the typical experimental geometry of a THz-streaking experiment [1, 3, 4]. Figure 1 (b) shows an example of a streaking spectrogram recorded upon ionization of Xe-Ar clusters with intense FEL pulses (92 eV photon energy) [3]. In this experiment $\mathrm{THz}$ radiation is produced in the $\mathrm{THz}-$ undulator by the same electron bunch that is used to produce XUV pulse [1]. Note that electrons released from the $4 \mathrm{~d}$ orbital of Xe show typical oscillatory behavior due to the shift in electron momentum induced by the instantaneous THz-field at the instance of the electron emission (Figure 1 (b)). Electrons marked as CED (correlated electronic decay), however, remain un-streaked, indicating that these electrons are emitted long after the $\mathrm{THz}$ pulse is terminated (i.e. on the time-scale of at least few picoseconds) [3].

For experiments at the MAC user end-station atomic, molecular, cluster or aerosol nano-particles will be available as targets. XUV pulses will be delivered by the HHG-source to the MAC end-station. Two types of THz-sources are planned. The first one is based on the optical rectification in a LiNbO3crystal. To achieve phase matching conditions a tilted-pulse-front geometry will be used $[5,6]$. This source typically provides single cycle $\mathrm{THz}$ pulses in the frequency range between 0.1 and $1 \mathrm{THz}$. The expected maximal $\mathrm{THz}$-field strength in the interaction region is $\sim 10 \mathrm{MV} / \mathrm{m}$ [4]. The second source of $\mathrm{THz}$ radiation is based on difference frequency generation in an organic crystal [7]. This source provides narrow band multi-cycle THz-pulse tunable in the frequency range between 4 and $18 \mathrm{THz}$. Peak electric field of $370 \mathrm{MV} / \mathrm{m}$ has been achieved [7]. Using the same near-infrared laser to drive the HHG process and the $\mathrm{THz}$-source will provide $\mathrm{XUV}$ and $\mathrm{THz}$-pulses that are intrinsically synchronized with sub-cycle accuracy [4]. These developments will enable advanced experiments on the structure and dynamics of matter using a combination of $\mathrm{THz}$ and $\mathrm{HHG}$ beams.

Acknowledgements: This work was supported by the projects Advanced research using high intensity laser produced photons and particles (ADONIS) (CZ.02.1.01/0.0/0.0/16_019/0000789) and Structural dynamics of biomolecular systems (ELIBIO) (CZ.02.1.01/0.0/0.0/15_003/ 0000447) from the European Regional Development Fund and the Ministry of Education, Youth and Sports as part of targeted support from the National Programme of Sustainability II.

\section{References}

1. Frühling, U.., Wieland M., Gensch, M., Gebert, T., Schütte, B., Krikunova,M., Kalms, K., Budzyn, F., Grimm, O., Rossbach, J., Plönjes, E., Drescher, M.. Single-shot terahertz-field-driven X-ray streak camera // Nature Photon. 2009. V. 3, P. 523-528.

2. Kienberger, R., Goulielmakis, E., Uiberacker, M., Baltuska, A., Yakovlev, V., Bammerr, F., Scrinzi, A., Westerwalbesloh, T., Kleineberg, U., Heizmann, U., Drescher, M., Krauz, F.. Atomic transient recorder // Nature, 2004. V. 427. P. 817-821.

3. Oelze, T., Schütte, B., Müller, M., Müller, J.P., Wieland, M., Frühling, U., Drescher, M., Al-Shemmary, A., Golz, T., Stojanovic, N., Krikunova, M. Correlated electronic decay in expanding clusters triggered by intense XUV pulses from a Free-Electron-Laser // Sci. Rep. 2017. V. 7, P. 40736.

4. Schütte, B., Frühling, U., Wieland, M., Azima, A., Drescher, M. Electron wave packet sampling with lasergenerated extreme ultraviolet and terahertz fields // Opt. Express 2011. V. 19, No. 20. P. 18833-18841.

5. Hebling, J., Almasi, G., Kozma, I.Z. Velocity matching by pulse front tilting for large-area THz-pulse generation // Geophys. Opt. Express 2002. V. 10, No. 21. P. 1161-1166. 6. Yeh, K.L., Hoffmann, M.C., Hebling, J., Nelson, K.A. Generation of $10 \mu \mathrm{J}$ ultrashort terahertz pulses by optical rectification // Appl. Phys. Lett. 2007. V. 90, No. 17. P. 171121.

7. Liu, B., Bromberger, H., Cartella, A., Gebert, T., Först, M., Cavalleri, A.. Generation of narrowband, high-intensity, carrier-envelope phase-stable pulses tunable between 4 and $18 \mathrm{THz} / /$ Opt. Lett. 2017. V. 42, No. 1. P. 129-131. 